\title{
Homotopy self-equivalences of 4-manifolds
}

\author{
Ian Hambleton • Matthias Kreck
}

(C) Springer-Verlag 2006

Erratum to: Math. Z. 248(1):147-172

DOI 10.1007/s00209-004-0657-9

\begin{abstract}
We correct an error in the calculation of the braid diagram of Hambleton and Kreck (Math. Z. 248, 147-172, 2004) for 4-manifolds with finite odd order fundamental group.
\end{abstract}

Our calculations of $[1$, Sect. 4$]$ contain a mistake: we implicitly assumed that

$$
\operatorname{Aut}_{\bullet}(B)=\operatorname{Isom}\left(\left[\pi_{1}(M), \pi_{2}(M), k_{M}\right]\right)
$$

where $B=B(M)$ denotes the 2-type of a closed, oriented, smooth or topological 4-manifold $M$ (see the proofs of Lemma 4.4 and Corollary 4.10, as well as the corresponding parts of the non-spin case on p. 170).

The homotopy self-equivalences of 2-stage Postnikov systems have been studied by a number of authors (see [2] and the references given there). The main result of Møller [2] applied to our special case is an exact sequence

$$
0 \rightarrow H^{2}\left(\pi_{1} ; \pi_{2}\right) \rightarrow \operatorname{Aut}_{\bullet}(B) \rightarrow \operatorname{Isom}\left(\left[\pi_{1}, \pi_{2}, k_{M}\right]\right) \rightarrow 1
$$

Research partially supported by NSERC Research Grant A4000.

The online version of the original article can be found at http://dx.doi.org/10.1007/s00209-004-0657-9.

I. Hambleton $(\bowtie)$

Department of Mathematics and Statistics, McMaster University,

Hamilton, ON L8S 4K1, Canada

e-mail: ian@math.mcmaster.ca

M. Kreck

Mathematisches Institut, Universität Heidelberg, 69120 Heidelberg, Germany

e-mail: kreck@mathi.uni-heidelberg.de 
whose extension class is related to the $k$-invariant of $B$. In order to correct the statements of our results, we define

$$
\widetilde{\operatorname{Isom}}\left(\left[\pi_{1}, \pi_{2}, k_{M}, s_{M}\right]\right)=\left\{\phi \in \operatorname{Aut}_{\bullet}(B) \mid \phi_{*}\left(c_{*}[M]\right)=c_{*}[M]\right\}
$$

This is the subgroup of Aut. $(B)$ fixing the image of the fundamental class of $M$ in $H_{4}(B ; \mathbf{Z})$. In the case $\pi_{1}(M)$ is finite of odd order, we obtain an exact sequence

$$
0 \rightarrow H^{5}\left(\pi_{1} ; \mathbf{Z}\right) \rightarrow \widetilde{\operatorname{Isom}}\left(\left[\pi_{1}, \pi_{2}, k_{M}, s_{M}\right]\right) \rightarrow \operatorname{Isom}\left(\left[\pi_{1}, \pi_{2}, k_{M}, s_{M}\right]\right) \rightarrow 0
$$

and this middle term is the image of Aut. $(M)$ in Aut॰ $(B)$. The calculation

$$
H^{2}\left(\pi_{1} ; \pi_{2}\right)=H^{5}\left(\pi_{1} ; \mathbf{Z}\right)
$$

is contained in the proof of Proposition 4.8. The statements of Lemma 4.4 and Corollary 4.10, together with Theorems A and B are now corrected by replacing the arithmetic group Isom( $\left.\left[\pi_{1}, \pi_{2}, k_{M}, s_{M}\right]\right)$ with its finite extension

$$
\widetilde{\operatorname{Isom}}\left(\left[\pi_{1}, \pi_{2}, k_{M}, s_{M}\right]\right) \text {. }
$$

We would also like to point out that the injectivity of the first map in the exact sequence the end of [1, p. 162] holds for odd order fundamental groups, but not in general.

\section{References}

1. Hambleton, I., Kreck, M.: Homotopy self-equivalences of 4-manifolds. Math. Z. 248, 147-172 (2004)

2. Møller, J.M.: Self-homotopy equivalences of group cohomology spaces. J. Pure Appl. Algebra 73, 23-37 (1991) 